\title{
The role of APOBEC3B in the development of hepatocellular carcinoma should be investigated with the consideration of hepatitis $B$ virus evolution
}

\author{
Wen-Bin Liu, Guang-Wen Cao \\ Department of Epidemiology, Second Military Medical University, Shanghai 200433, China.
}

Correspondence to: Prof. Guang-Wen Cao, Department of Epidemiology, Second Military Medical University, 800 Xiangyin Rd, Shanghai 200433, China.E-mail: gcao@smmu.edu.cn

\begin{abstract}
How to cite this article: Liu WB, Cao GW. The role of APOBEC3B in the development of hepatocellular carcinoma should be investigated with the consideration of hepatitis B virus evolution. Hepatoma Res 2019;5:30.

http://dx.doi.org/10.20517/2394-5079.2019.27
\end{abstract}

Received: 16 Jul 2019 First Decision: 17 Jul 2019 Revised: 17 Jul 2019 Accepted: 19 Jul 2019 Published: 7 Aug 2019

Science Editor: Guang-Wen Cao Copy Editor: Jia-Jia Meng Production Editor: Jing Yu

The chronic infection of hepatitis B virus (HBV) is the major cause of hepatocellular carcinoma (HCC) globally $^{[1]}$. In Eastern China, chronic HBV infection contributes to $87.5 \%$ of HCC whereas chronic hepatitis $\mathrm{C}$ virus (HCV) infection contributes to $1.7 \%^{[2]}$. The mortality of HCC has increased in Europe and America over recent decades ${ }^{[3]}$. Although the infection of HCV is the leading cause of HCC in most European and American countries, the contribution of HBV is increasing possibly due to immigration ${ }^{[3]}$.

HCC represents a typical paradigm of inflammation-cancer transformation. Based on the advances in HBVinduced hepatocarcinogenesis, a scientific theory of Cancer Evolution-Development (Cancer Evo-Dev) was proposed $^{[4-6]}$. The central aspects of this theory include: the interaction of HBV infection and immunogenetic predispositions maintains non-resolving inflammation. Immune imbalance promotes the generation of somatic and viral mutations via disbalancing Apolipoprotein B mRNA-editing enzyme catalytic polypeptide-like 3B (АРОВЕСзВ) and mutation-repairing forces. Most mutant cells are eliminated by inflammatory microenvironment while only a small percentage of cells adapt the environment and survive. These survived mutant clones evolve to tumor-initiating cells (TICs) by altering the signal patterns mainly caused by de-differentiation mechanisms. TICs acquire the stemness and the ability of immune escape through recruiting tumor-associated macrophages (TAM) and myeloid-derived suppressor cells (MDSCs). Under the pressure of selection, TICs further obtain metastatic and drug-resistant potentials to adapt to distinct microenvironments. The evolution of HBV occurs along with this process. The mutant virus that selected by inflammatory environment can survive the immune elimination and facilitate the malignant

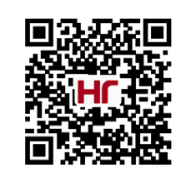


transformation of normal cells. Thus, the HCC development is characterized by an evolutionary process of mutation-selection-adaptation.

APOBEC3B can generate cytosine-to-uracil $(\mathrm{C}>\mathrm{U})$ transversions through deamination. APOBEC $3 \mathrm{~B}$ related mutation pattern is proved to be widespread in the genome of tumors in many different organs including breast, lung, cervix, ovary, bladder, and head and neck ${ }^{[7]}$. Therefore, APOBEC 3 B is commonly believed to be a major force of generating somatic mutations.

In a recent study, Wang et al. ${ }^{[8]}$ revealed another important role of APOBEC3B in the cancer evolutionary process of mutation-selection-adaptation. Their study suggests that APOBEC3B can also contribute to the "selection" and "adaption" of malignant cells via facilitating the immune escape in a deaminaseindependent way. They reported that the elevated abundance of AРОВЕСзB in HCC predicts a poor prognosis. Authors demonstrated a high affinity $\kappa \mathrm{B}$ site in APOBECзB promoter and non-canonical NF$\kappa \mathrm{B}$ signaling pathway up-regulates the expression of $\mathrm{APOBEC} 3 \mathrm{~B}$ via activating its transcription. With the animal models of HCC, it was demonstrated that elevated APOBEC3B facilitated the development of HCC only in the immunocompetent mouse rather than in the immune-deficient mouse. APOBEC3B was proved to recruit TAM, MDSC, and $\mathrm{CD}^{+} \mathrm{T}$ cells positive for Programmed cell death ligand 1 through increasing the secretion of $\mathrm{C}-\mathrm{C}$ motif chemokine ligand 2 (CCL2). The immunosuppressive effect of APOBEC3B depends on epigenetic modification. $\mathrm{APOBEC} 3 \mathrm{~B}$ can inhibit the activity of polycomb repressor complex 2, which is essential for maintaining methylation of $\mathrm{H}_{3} \mathrm{~K} 27$. Therefore, the elevated APOBEC3B in HCC depresses global $\mathrm{H} 3 \mathrm{~K} 27 \mathrm{me} 3$ abundance and reduces the occupancy of $\mathrm{H} 3 \mathrm{~K} 27 \mathrm{me} 3$ on the promoter of CCL2. Thus, APOBEC3B promotes the immune escape and growth of HCC.

This remarkable study highlights the role of $\mathrm{APOBЕСзB} \mathrm{in} \mathrm{regulation} \mathrm{of} \mathrm{immune} \mathrm{microenvironment} \mathrm{as} \mathrm{a}$ factor of epigenetic modification. The understanding of APOBEC3B function and the theory of Cancer EvoDev are improved due to their solid evidences. In the meantime, two questions are proposed in this article. First, in addition to regulating epigenetic modification, APOBECзB can promote $\mathrm{HCC}$ development by inducing mutation. Second, the role of interaction between HBV and APOBEC3B-mediated inflammatory microenvironment in HCC evolution should be further investigated. In this study, HBV infection was not taken into consideration. The immunosuppressive function of $\mathrm{APOBEC} 3 \mathrm{~B}$ was mainly demonstrated with the diethylnitrosamine-induced HCC animal model, which can hardly reflect the HBV-induced carcinogenesis in human. As described in the article, what authors investigated is the "hepatoma-intrinsic АРОВЕСзB" rather than $\mathrm{APOBEC} 3 \mathrm{~B}$ of hepatocytes with chronic inflammation. Therefore, results of this study cannot represent all the effects of APOBEC $3 \mathrm{~B}$ during HBV-induced hepatocarcinogenesis, especially its mutagenic function. $\mathrm{APOBEC} 3 \mathrm{~B}$ contributes the innate immune responses to $\mathrm{HBV}$ infection through inhibiting the replication of HBV via hyper-editing viral genome ${ }^{[9]}$. Although APOBEC3B induced HBV mutations are highly deleterious, a small percentage of viral mutations can facilitate the immune escape or the regeneration of hepatocytes ${ }^{[5]}$.

Interestingly, another recent study revealed the role of APOBEC 3B in HCC development from another aspect, which answers the above questions. It discovered the associations among genetic predispositions, inflammation, АРОВЕС 3 B, and HBV mutations during the process of HCC development ${ }^{[10]}$. Interleukin- 6 (IL-6) was proved to increase the expression of $\mathrm{APOBEC} 3 \mathrm{~B}$ and decrease the expression of uracil DNA glycosylase (UNG), an enzyme essential for DNA repair, thus leading to imbalance of mutagenic forces and mutation-repairing forces. Two genetic polymorphisms, rs2267401 (G) and rs3890995 (C), were proved to intensify the IL-6 induced APOBEC3B-UNG imbalance through affecting the activities of APOBEC3B promoter and UNG enhancer, respectively. These two genetic polymorphisms were also proved to be significantly associated with increased HCC risk by using a large case-control study involving 5221 participants. Besides, variant genotypes at rs2267401 were also demonstrated to improve the accumulation 
of APOBEC3-signature HBV mutations and A1762T/G1764A in HBV-infected subjects, both of which were confirmed to associated with increased risk of HCC. The data of cohort studies demonstrated that APOBEСзB rs2267401-GG genotype, higher APOBEC3B expression, and higher APOBEC3B/UNG expression ratio in HCCs can predict a poor prognosis. Interestingly, APOBEC-signature somatic mutation predicts poor prognosis only in HBV-free HCC rather than in HBV-positive ones. These evidences strongly suggest that $\mathrm{APOBEC} 3 \mathrm{~B}$ facilitates $\mathrm{HBV}$-induced $\mathrm{HCC}$ evolution via its mutagenic effect preferentially on the HBV genome. This result also explains why the APOBEC3-signature somatic mutation was not dominant in HCC genome ${ }^{[11]}$. APOBEC3B prefers to edit HBV genome possibly because the number of HBV genomic DNA is overwhelmingly more than that of human genome. Besides, during the replication of HBV, the partially double-stranded HBV DNA is generated from an intermediate RNA that is vulnerable to $\mathrm{APOBEC} 3 \mathrm{~B}$.

To conclude, the work by Wang and related studies demonstrated the important role of APOBEC3B in $\mathrm{HCC}$ evolution from different aspects. APOBEC3B promotes $\mathrm{HBV}$-induced carcinogenesis through its mutagenic activity and facilitating immune escape of HCC through regulating epigenetic modification. The investigation for APOBEС $3 \mathrm{~B}$ can be transformed not only into specific prophylaxis but also into target therapy.

\section{DECLARATIONS}

\section{Authors' contributions}

Study concept and design: Cao GW

Drafting of the manuscript: Liu WB

Discussion and revision of the manuscript text: Cao GW

\section{Availability of Data and Materials}

Not applicable.

\section{Financial support and sponsorship}

This work was supported by grant (2015CB554006) from the National Key Basic Research Program of China (GC).

\section{Conflicts of interest}

All authors declared that there are no conflicts of interest.

\section{Ethical Approval and Consent to Participate}

Not applicable.

\section{Consent for publication}

Not applicable.

\section{Copyright}

(c) The Author(s) 2019.

\section{REFERENCES}

1. Sia D, Villanueva A, Friedman SL, Llovet JM. Liver cancer cell of origin,molecular class, and effects on patient prognosis. Gastroenterology 2017;152:745-61.

2. Yang F, Ma L, Yang Y, Liu W, Zhao J, et al. Contribution of Hepatitis B Virus Infection to the Aggressiveness of Primary Liver Cancer: A Clinical Epidemiological Study in Eastern China. Front Oncol 2019;21;9:370.

3. Bertuccio P, Turati F, Carioli G, Rodriguez T, La Vecchia C, et al. Global trends and predictions in hepatocellular carcinoma mortality. 
J Hepatol 2017;302-9.

4. Liu W, Wu J, Du Y, Cao G. Cancer Evolution-Development: experience of hepatitis B virus-induced hepatocarcinogenesis. Curr Oncol 2016;23:e49-56.

5. Deng Y, Du Y, Zhang Q, Han X, Cao G. Human cytidine deaminases facilitate hepatitis B virus evolution and link inflammation and hepatocellular carcinoma. Cancer Lett 2014;343:161-71.

6. Cao G. Cancer Evo-Dev, a novel hypothesis derived from studies on hepatitis B virus-induced carcinogenesis. Hepatoma Res 2017;3:241-59.

7. Roberts SA, Lawrence MS, Klimczak LJ, Grimm SA, Fargo D, et al. An APOBEC cytidine deaminase mutagenesis pattern is widespread in human cancers. Nat Genet 2013;45:970-6.

8. Wang D, Li X, Li J, Lu Y, Zhao S, et al. APOBEC3B interaction with PRC2 modulates microenvironment to promote HCC progression. Gut 2019. Epub ahead of print. doi: 10.1136/gutjnl-2018-317601.

9. Lucifora J, Xia Y, Reisinger F, Zhang K, Stadler D, et al. Specific and nonhepatotoxic degradation of nuclear hepatitis B virus cccDNA. Science 2014;343:1221-8.

10. Liu W, Wu J, Yang F, Ma L, Ni C, et al. Genetic polymorphisms predisposing the interleukin-6-induced APOBEC3B-UNG imbalance increase HCC risk via promoting the generation of APOBEC-signature HBV mutations. Clin Cancer Res 2019. Epub ahead of print. doi: 10.1158/1078-0432.CCR-18-3083

11. Alexandrov LB, Nik-Zainal S, Wedge DC, Aparicio SA, Behjati S, et al. Signatures of mutational processes in human cancer. Nature 2013;500:415-21. 